\title{
A comparison between structural equation modelling (SEM) and Bayesian SEM approaches on in-store behaviour
}

\begin{abstract}
Purpose

The purpose of this paper is to examine the effects of atmospherics and affective state on shoppers' in-store behaviour using the two approaches in structural equation modelling (SEM), i.e. Frequentist and Bayesian approaches. Shoppers' affective state was tested for its mediating effect on in-store shopping behaviour.

Design/methodology/approach

The final sample consists of 382 respondents who were drawn from shoppers at selected apparel stores in six of the most popular shopping malls around Kuala Lumpur (Malaysia). A frequentist approach to SEM is common among researchers and offers generally an analysis of the relationships between multiple latent variables and constructs. Alternatively, the Bayesian SEM (BSEM) approach stems from the diffusion of the model's posterior distributions using the Markov Chain Monte Carlo technique. More specifically, this technique is inherently more flexible and substantive in determining parameter estimates as compared to the more conventional, the frequentist approach to SEM.
\end{abstract}

Findings

The results show the mixed effects of atmospheric cues in retail setting on shoppers' affective state. More specifically, the positive direct effect of atmospheric cues (music) on in-store behaviour was confirmed while other atmospheric cues (colour and store layout) were found to be fully mediated by affective state. The Bayesian approach was able to offer more distinctive results complementing the frequentist approach.

Research limitations/implications

Although the current sample size is adequate, it will be interesting to examine how a bigger sample size and different antecedents of in-store behaviour in retailing can affect the comparison between the frequentist approach in SEM and BSEM.

Practical implications

The authors found that a combination of well-designed store atmospherics and layout store can produce pleasurable effects on shoppers resulting in positive affective state. This study found that results from both frequentist and Bayesian approaches complement each other and it may be beneficial for future studies to utilise both approaches in SEM. 
Originality/value

This paper met the aim to compare the approaches in SEM and the need to consider both approaches on in-store shopping environment. Overall, the authors contend that the Bayesian approach to SEM is a potentially viable alternative to frequentist SEM, especially when studies are conducted under dynamic conditions such as apparel retailing.

Keyword: Atmospherics; Affective state; Bayesian SEM; Frequentist structural equation modelling; In-store behaviour; Markov Chain Monte Carlo 